\title{
Pseudochylothorax in pleural effusion due to coronary artery bypass surgery
}

\author{
E. Garcia-Pachon*, L.C. Fernandez*, F. Lopez-Azorin**, I. Padilla-Navas ${ }^{+}$
}

\begin{abstract}
Pseudochylothorax in pleural effusion due to coronary artery bypass surgery. E. GarciaPachon, L.C. Fernandez, F. Lopez-Azorin, I. Padilla-Navas. (C) ERS Journals Ltd 1999.

ABSTRACT: This study describes a 64-yr-old male with a chronic left pleural effusion following a coronary artery bypass 3 yrs earlier. On thoracocentesis, turbid fluid was obtained with crystals of cholesterol on microscopic examination, establishing the diagnosis of pseudochylothorax. The pleural fluid cholesterol level was $207 \mathbf{~ m g} \cdot \mathbf{d L}^{-1}$ $\left(5.36 \mathrm{mmol} \cdot \mathrm{L}^{-1}\right)$. This is the first report of pseudochylothorax in a chronic pleural effusion due to coronary artery bypass surgery.

Eur Respir J 1999; 13: 1487-1488.
\end{abstract}

*Dept of Medicine and **Laboratory Service, Hospital Vega Baja, Orihuela-Alicante, Spain. ${ }^{+}$Respiratory Medicine Section, Hospital General Universitari d'Elx, Alicante, Spain.

Correspondence: E. Garcia-Pachon, Dept of Medicine, Hospital Vega Baja, E-03314 Orihuela-Alicante, Spain. Fax: 3496677 6060 .

Keywords: Coronary artery bypass surgery, pleural effusion, pseudochylothorax

Received: April 231998

Accepted after revision September 91998
Pseudochylothorax is a pleural effusion with a very high content of cholesterol [1]. It can occur when fluid has been present in the pleural space for a long time especially with fibrotic pleura $[1,2]$. The three most common causes of pseudochylothorax are tuberculous pleural effusion, therapeutic pneumothorax, and chronic rheumatoid pleurisy [1-4], but it can develop with other diseases which cause extensive fibrosis of the pleura [1]. Chronic pleural effusion and pleural thickening may occur following coronary artery bypass, but this procedure has not previously been described as a cause of pseudochylothorax.

\section{Case report}

A 64-year-old male with a past medical history of myocardial infarction and coronary artery bypass surgery was admitted to the authors' hospital because of a decompensation of congestive heart failure which resolved in a few days following conventional therapy. Chest radiographs during the admission showed an unchanging pleural effusion that occupied more than one-third of the left hemithorax. The patient was aware that he had a chronic pleural effusion after having undergone coronary artery bypass 3 yrs earlier at another hospital. The radiograph prior to surgery was normal and he had no history of previous pleural disease, tuberculosis, or rheumatoid arthritis. The cardiac surgeon followed the patient for $1 \mathrm{yr}$ and informed him that he had a left pleural effusion which had not changed during this period. At that time, no thoracocentesis or other diagnostic tests were performed. On admission to our hospital, a chest computed tomographic scan showed the large left pleural effusion with thickened pleura and a small right pleural effusion. Thoracocentesis was performed and turbid fluid was obtained. Examination for malignant cells was negative, as were stains and cultures for bacteria, mycobacteria and fungi. Pleural fluid analysis showed an exudative effusion with a cholesterol level of $207 \mathrm{mg} \cdot \mathrm{dL}^{-1}\left(5.36 \mathrm{mmol} \cdot \mathrm{L}^{-1}\right)$ and triglycerides $81 \mathrm{mg} \cdot \mathrm{dL}^{-1}$ $\left(0.90 \mathrm{mmol} \cdot \mathrm{L}^{-1}\right)$. Crystals of cholesterol were seen on microscopic examination, confirming the diagnosis of pseudochylothorax. The patient declined to undergo pleural decortication.

\section{Discussion}

The frequency of pleural effusion following coronary artery bypass graft surgery is very high. The effusion tends to be unilateral on the left side and is usually small $[5,6]$. Since most patients have pleural effusion after coronary artery bypass surgery, diagnostic thoracocentesis is not usually warranted [6]. Recurrent or chronic pleural effusions and pleural fibrosis are also recognized following coronary artery revascularization $[7,8]$. Some exudates which persist for a long time (months or years) in the pleural space have a tendency to become enriched with cholesterol. Typically, this cholesterol pleurisy is seen in fluid which is encapsulated within grossly thickened pleura [1]. Since a chronic pleural effusion within fibrotic pleura is possible after coronary artery bypass surgery, it is reasonable to expect that this may result in a pseudochylothorax. Nevertheless, such as association has not previously been reported, although in the series of KOLLEF et al. [7] the pleural fluid in one case was described as dark amber coloured and in another patient pleural thickening was present on the chest radiograph; the cholesterol level in the pleural fluid was not determined. Because coronary artery bypass surgery is performed very frequently and chronic pleural effusion is a well-known 
complication of this technique, it may become one of the more common causes of pseudochylothorax, especially since classical causes (such as chronic tuberculous pleurisy or therapeutic pneumothorax) are becoming increasingly rare [1].

\section{References}

1. Hillerdal G. Chylothorax and pseudochylothorax. Eur Respir J 1997; 10: 1157-1162.

2. Coe JE, Aikawa JK. Cholesterol pleural effusion. Arch Intern Med 1961; 108: 763-774.

3. Ferguson CG. Cholesterol pleural effusion in rheumatoid lung disease. Thorax 1966; 21: 577-582.
4. Hillerdal G. Chyliform (cholesterol) pleural effusion. Chest 1985; 88: 426-428.

5. Vargas FS, Cukier A, Hueb W, Teixeira LR, Light RW. Relationship between pleural effusion and pericardial involvement after myocardial revascularization. Chest 1994; 105: 1748-1752.

6. Light RW. Pleural effusion due to miscellaneous diseases. In: Light RW, ed. Pleural Diseases. Baltimore, Williams \& Wilkins, 1995; pp. 224-241.

7. Kollef MH, Peller T, Knodel A, Cragun WH. Delayed pleuropulmonary complications following coronary artery revascularization with the internal mammary artery. Chest 1988; 94: 68-71.

8. Kollef MH. Trapped-lung syndrome after cardiac surgery: a potentially preventable complication of pleural injury. Heart Lung 1990; 19: 671-675. 